\title{
The Influence of Verbal Cues on Drop Jump Landing Strategies in NCAA Division I Soccer Players
}

\author{
Leland Barker, *, Scott Bankers², Brooke Farmer ${ }^{3}$, Jake Siedlik ${ }^{1}$, John Harry ${ }^{4}$, \\ Terry L. Grindstaff ${ }^{3}$ \\ ${ }^{1}$ Department of Exercise Science \& Pre-Health Professions, Creighton University, Omaha, Nebraska, The United States \\ ${ }^{2}$ Department of Athletics, Creighton University, Omaha, Nebraska, The United States \\ ${ }^{3}$ Department of Physical Therapy, Creighton University, Omaha, Nebraska, The United States \\ ${ }^{4}$ Department of Kinesiology and Sport Management, Texas Tech University, Lubbock, Texas, The United States
}

Email address:

lab58411@creighton.edu (L. Barker)

${ }^{*}$ Corresponding author

\section{To cite this article:}

Leland Barker, Scott Bankers, Brooke Farmer, Jake Siedlik, John Harry, Terry L. Grindstaff. The Influence of Verbal Cues on Drop Jump Landing Strategies in NCAA Division I Soccer Players. American Journal of Sports Science. Vol. 9, No. 2, 2021, pp. 37-42. doi: $10.11648 /$ j.ajss.20210902.12

Received: May 4, 2021; Accepted: May 19, 2021; Published: May 27, 2021

\begin{abstract}
Jump testing to monitor athletic performance and development has become commonplace in the field of strength and conditioning. Verbal cues of jump tests, such as the drop jump, has significant effects on performance metrics and movement strategy. The purpose of this study was to use a force platform analysis to investigate landing depth and global flexion in the lower extremity joints at initial impact between cues to maximize jump height or minimize ground contact time. Another purpose of this study was to repeat previous investigations on the influence of cueing DVJs to maximize height or minimize ground contact time on the Reactive Strength Index (RSI). Thirty-nine Division I soccer players performed one DVJ $\left(\mathrm{DVJ}_{\text {Height }}\right)$ for maximum height and another DVJ $\left(\mathrm{DVJ}_{\text {Quick }}\right)$ for quickness. Differences in dependent variables were analyzed

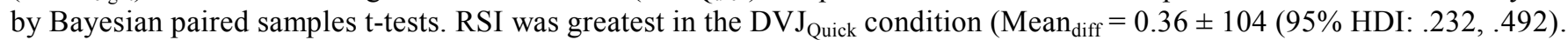
Ground contact time was shorter with the $\mathrm{DVJ}_{\text {Quick }}\left(\mathrm{Mean}_{\text {diff }}=-0.19 \pm 0.028,95 \%\right.$ HDI: $\left.-0.224,-0.155\right)$. Jump height was less with the $\mathrm{DVJ}_{\text {Quick }}\left(\mathrm{Mean}_{\text {diff }}=-0.033 \pm 0.007,95 \%\right.$ HDI: $\left.-0.068, .0035\right)$. Landing depth decreased during the $\mathrm{DVJ}_{\text {Quick }}\left(\mathrm{Mean}_{\text {diff }}\right.$ $=-0.093 \pm 0.018,95 \%$ HDI: $-0.118,-0.066)$. Global flexion in the lower extremities at impact decreased with a DVJ $\mathrm{Quick}$ $\left(\mathrm{Mean}_{\text {diff }}=-0.057 \pm 0.025,95 \%\right.$ HDI: $\left.-0.084,-0.031\right) . \mathrm{DVJ}_{\text {Quick }}$ resulted in a large increase in RSI due to disproportionate decreases in ground contact time (large decrease) compared to jump height (small decrease). The increase in RSI with $\mathrm{DVJ}_{\text {Quick }}$ coincided with a decrease in landing depth, suggesting a stiff landing strategy and increased stretch-shortening cycle intensity.
\end{abstract}

Keywords: Drop Vertical Jump, Strategies, Stiffness

\section{Introduction}

Jump testing to monitor athletic performance and development has become commonplace in the field of strength and conditioning. Monitoring jump performance can be used to assess training program efficacy $[18,5]$, return-to-play decisions following injury [11], fatigue [19], athlete workload monitoring [26], and predict athletic success [7, 9]. A common jump test is the drop vertical jump (DVJ), where an athlete steps off a box and attempts to jump as high as possible after landing. The DVJ is particularly suited for testing stretch-shortening cycle performance of the lower extremity [18].

One common metric to assess DVJ performance is the Reactive Strength Index (RSI). RSI is calculated as jump height divided by ground contact time during a DVJ, making it a time-sensitive measure of performance. RSI has been shown to be both reliable ( $\mathrm{ICC}=0.90, \mathrm{CV}=5 \%)$ [3] and associated with maximum strength $(\mathrm{r}=0.302-0.431)$ [2], sprinting ability $(\mathrm{RSI}=3.02$ in sprint-trained athletes compared to $\mathrm{RSI}=2.02$ 
in non-sprint-trained recreational athletes) [9], eccentric force production (Eccentric Rate of Force Development, $r=0.755$ ) [1], and running economy $(\mathrm{r}=-0.419--0.572)$ [21]. To date, a few studies have investigated how landing technique influences RSI. One recent study of youth soccer athletes reported increased RSI when the DVJ is cued to minimize ground contact time, and decreased RSI when cued for maximal height [23]. Verbal cues have also been reported to influence stiffness in drop jumps [17], and force-velocity-power profiles during countermovement jumps [24]. In a general population sample, DVJ RSI was similar using self-selected $\left(0.96 \pm 0.4\right.$; Knee flexion: $\left.89.5 \pm 14.6^{\circ}\right)$ and stiff (RSI: $0.93 \pm 0.5$; Knee flexion: $60.4 \pm 13.1^{\circ}$ ) landing techniques, which were greater than a soft (RSI: $0.64 \pm 0.3$; Knee flexion: $116.1 \pm 11.1^{\circ}$ ) landing technique [12]. Landing depth appears to be the primary difference between these techniques according to the range of knee flexion angles across self-selected, stiff, and soft landing techniques [12]. Given the changes in RSI between stiff and soft landing techniques, these reports indicate disproportionate changes in jump height and ground contact time when landing depth varies. We suspect elite athletes may be able to generate a greater range of RSI values across landing depths than the general population.

There may be a landing depth that maximizes RSI for a given athlete despite no real changes in their jumping ability (i.e. they improve their technique rather than improving force production with consistent technique). If landing depth is free to vary or not accounted for, it is impossible to determine whether reductions in ground contact time or increases in jump height occurred due to manipulations of landing depth and stiffness or improvements in force production. Therefore, the consideration of landing depth is critical to observe real and meaningful changes in DVJ performance if practitioners are using RSI to test athletic development over time. In appreciation for the variable demands of sporting competition, it may be necessary for practitioners to test multiple DVJ techniques since various landing depths and stiffness qualities influence lower extremity joint kinetics and energetics [8].

Cueing DVJs to either maximize jump height, minimize ground contact time, or both may be a practical method to investigate differences in landing strategies and its influence on RSI and landing mechanics. For example, increasing landing depths could increase work capacity of the lower extremity joints to increase jump height and ground contact time, whereas decreasing landing depth could decrease work capacity of the lower extremity to decrease ground contact time and jump height. If ground contact time decreases proportionately with decreases in jump height, RSI would remain unchanged. It is not clear how verbal cues to maximize jump height or minimize ground contact time would influence landing strategies and technique. For example, the amount of lower extremity joint flexion at impact is free to vary in addition to the landing depth during deceleration. Previous research has reported lower-body flexion at the hip, knee, and ankle occurs prior to initial impact, which may differ when landing from a jump [13] than from a box [16, 25]. Therefore, the purpose of this study was to use a force platform analysis to investigate landing depth and global flexion in the lower extremity joints at initial impact between cues to maximize jump height or minimize ground contact time. We hypothesized the landing depth and amount of global flexion at impact would be greater in the DVJ cued for maximal height. Another purpose of this study was to repeat previous investigations on the influence of cueing DVJs to maximize height or minimize ground contact time on RSI. We hypothesized that an increase in RSI would occur with a cue to minimize ground contact time compared to a cue to maximize jump height due to a disproportionate decrease in ground contact time than jump height.

\section{Methods}

\subsection{Experimental Approach}

This study took place prior to an offseason training session and data collection occurred in a collegiate weight room setting. Due to time constraints, it was only possible for participants to complete a single maximum effort DVJ trial with two different verbal cues. One DVJ style was from a $0.305 \mathrm{~m}$ box. Each trial was cued to be performed "to jump as high as you can after landing" or "to get off the ground as quick as you can". Dependent variables analyzed from raw ground reaction force data (RSI, jump height, jump time, landing depth, global flexion at impact) were compared between cues.

\subsection{Subjects}

Thirty-nine Division I soccer players (19 women, 20 men, $69.3 \pm 9.9 \mathrm{~kg}, 1.74 \pm 0.1 \mathrm{~m}, 19.7 \pm 1.5$ years) participated in the study. All participants were active players on the university soccer team and were currently free of injury that limited participation in normal weight room activities. Subjects were informed of the benefits and risks of the investigation prior to signing informed consent approved by the XXXX university Institutional Review Board (No. $\mathrm{XXXXX)}$ as part of a larger collaboration with the athletic department.

\subsection{Procedures}

All trials were performed on a dual force-platform setup (Bertec Corporation, Columbus, $\mathrm{OH}$ ) interfaced with data acquisition software $(1000 \mathrm{~Hz}$; ForceDecks, Vald Performance) that allowed raw data to be exported for further analysis. Subjects performed testing prior to their regularly scheduled offseason training session. Due to time constraints, subjects performed only 1 maximal effort DVJ with each verbal cue following their normal warmup prior to training. Although 1 trial could be considered a limitation, subjects were given multiple attempts at a "good" trial and regularly performed the DVJ in their training programs. Subject body weight was recorded at the beginning of each trial where subjects stood still on the force platforms for 1-2 seconds. Next, the subject stepped onto the box $(30.5 \mathrm{~cm}$ box height, 
placed $15.25 \mathrm{~cm}$ from the force plates), stepped off, and performed the DVJ. One DVJ for maximal height $\left(\mathrm{DVJ}_{\text {Height }}\right)$ was cued "to jump as high as you can after landing", the other DVJ for quickness $\left(\mathrm{DVJ}_{\text {Quick }}\right)$ was cued "to get off the ground as quick as you can". Since stepping off a box can lead to some variability in actual drop height we calculated drop height from integration of vertical ground reaction force (vGRF) data to be $0.242 \pm 0.046 \mathrm{~m}$ in the $\mathrm{DVJ}_{\text {Height }}$ and $0.247 \pm 0.036 \mathrm{~m}$ in the $\mathrm{DVJ}_{\text {Quick}}$, which resulted in a small effect size of 0.128 . A rest period of 15-30 seconds was allowed between individual trials. Trials were repeated if the subject lost their balance, did not land completely on the force platforms, or perceived a lack of maximum effort.

\subsection{Data Analysis}

Raw vGRF data from all trials were exported to a spreadsheet (Microsoft Excel) and analyzed with a custom program (Matlab v2019a, Mathworks, Natick, MA). Based on recent reports looking at signal filtering during jumping [14], we chose to retain the raw data and not filter the signal.

Participant bodyweight was measured on a per trial basis from the average vGRF during the standstill on the force plate prior to stepping on to the box. The duration of the average was manually selected based on visual inspection of the trial, resulting in 1 second of quiet standing vGRF. vGRF data were used to calculate acceleration (acceleration $=(\mathrm{vGRF}-$ Bodyweight $(\mathrm{N})) /$ mass $(\mathrm{kg})$ ). Acceleration was then integrated with respect to time using the cumulative trapezoidal method to calculate velocity. Initial impact after leaving the box was labeled as the first frame when vGRF surpassed $20 \mathrm{~N}$, takeoff was labeled as the first frame when vGRF was less than $20 \mathrm{~N}$, and final landing was labeled as the first frame after takeoff that vGRF surpassed $20 \mathrm{~N}$. Time in the air was used to calculate jump height [jump height $=1 / 2(9.81)($ time in the air $)^{2}$. Figure 1 provides a visual representation of the calculations. RSI was calculated as jump height divided by ground contact time from impact to takeoff.

Time in the air was used to correct the integrated velocity values by setting vertical velocity equal to zero at the midpoint of flight phase. Velocity was then integrated with respect to time using the cumulative trapezoidal method to calculate displacement of the center of mass (COM). The initial position of the COM was set to 0 at impact. The global flexion in the lower extremity at impact was calculated as the COM displacement from impact to takeoff. Takeoff is assumed to occur with the participants in a fully extended position (i.e. 'triple extension'), thus global flexion at impact would be zero if impact position was the same as takeoff position (Figure 1) - across the entire study data we observed the minimum global flexion at impact to be $0.015 \mathrm{~m}$ for a single trial. Landing depth was calculated as the lowest COM position during ground contact in relation to the $\mathrm{COM}$ position at impact. Landing depth, therefore, is the vertical displacement required to decelerate the COM to zero velocity.

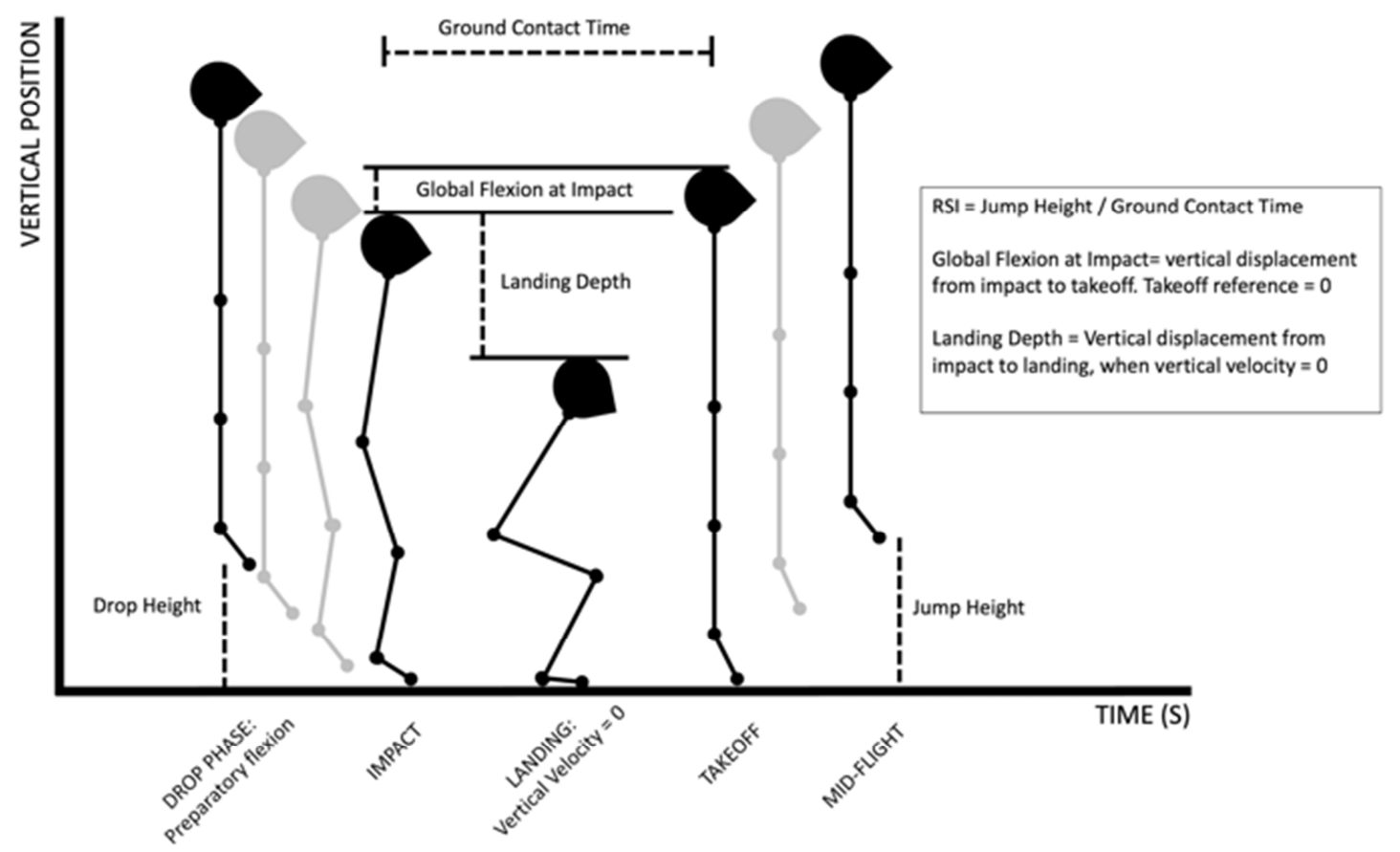

Figure 1. Visual representation of dependent variable calculations.

\subsection{Statistical Analysis}

Differences in dependent variables across the cueing conditions were analyzed by Bayesian paired samples t-tests using the BEST package in R $[15,20]$. Normality of data was verified using the D'Agostino-Pearson test and a non-informative, uniform prior was used for all analyses. Reported parameter estimates from Bayesian models include the posterior mean difference, 95\% highest density intervals (HDI), and effect sizes. Parameter estimates were interpreted as statistically significant if the $95 \%$ HDI did not include zero. 
All statistical analyses were performed using $\mathrm{R}$ version 3.6.1 [27].

\section{Results}

RSI was greatest in the $\mathrm{DVJ}_{\text {Quick }}$ condition with an estimated posterior $\mathrm{Mean}_{\text {diff }}=0.36 \pm 104 \quad(95 \%$ HDI:.232,.492). The 95\% HDI of the difference of the means does not include zero; therefore, there is a strong probability that the estimated parameter is greater than zero with an effect size estimate of 1.25 (95\% HDI: $0.773,1.8)$. In confirmation of our hypothesis, the increase in RSI occurred due to a disproportionate decrease in ground contact time compared to jump height. Ground contact time was 0.190 seconds shorter with the $\mathrm{DVJ}_{\text {Quick }}$ than $\mathrm{DVJ}_{\text {Height }}\left(\mathrm{Mean}_{\text {diff }}=-0.19 \pm 0.028,95 \%\right.$ HDI: $-0.224,-0.155)$ with an effect size estimate of $-2.62(95 \%$ HDI: -3.38, -1.88). Meanwhile, jump height during the $\mathrm{DVJ}_{\text {Quick }}$ was only $0.033 \mathrm{~m}$ less than the $\mathrm{DVJ}_{\text {Height }}\left(\mathrm{Mean}_{\text {diff }}=\right.$ $-0.033 \pm 0.007,95 \%$ HDI: $-0.068, .0035)$. The $95 \%$ HDI of the difference of the means includes zero but has $96.4 \%$ of the credible values less than zero. Thus, there is a strong probability $(96.4 \%)$ that the estimated parameter is less than zero (i.e. indicative of a decrease in jump height) with an effect size estimate of -0.41 (95\% HDI: $-0.882,0.042)$. With regard to landing technique and strategy to accomplish these differences in performance, we hypothesized global flexion at impact and landing depth to decrease with the $\mathrm{DVJ}_{\text {Quick. This }}$ hypothesis was confirmed by a $0.093 \mathrm{~m}$ decrease in landing depth during the $\mathrm{DVJ}_{\text {Quick }}$ compared to the $\mathrm{DVJ}_{\text {Height }}\left(\mathrm{Mean}_{\text {diff }}\right.$ $=-0.093 \pm 0.018,95 \%$ HDI: $-0.118,-0.066)$ with an effect size estimate of -1.69 (95\% HDI: $-2.27,-1.13)$. Global flexion at impact, which was measured as the vertical displacement between takeoff and initial landing, was $0.057 \mathrm{~m}$ less with a $\mathrm{DVJ}_{\text {Quick }}$ compared to the $\mathrm{DVJ}_{\text {Height }}\left(\mathrm{Mean}_{\text {diff }}=-0.057 \pm 0.025\right.$, 95\% HDI: $-0.084,-0.031)$ with an effect size estimate of -1.01 (95\% HDI: $-1.53,-0.514)$. This indicates the lower extremity joints were more extended (i.e., stiffer landing) at impact with a $\mathrm{DVJ}_{\text {Quick. }}$ a.

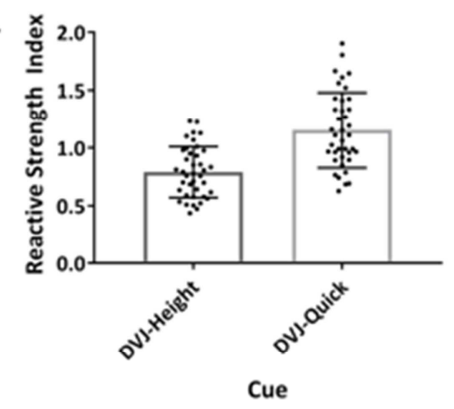

d.

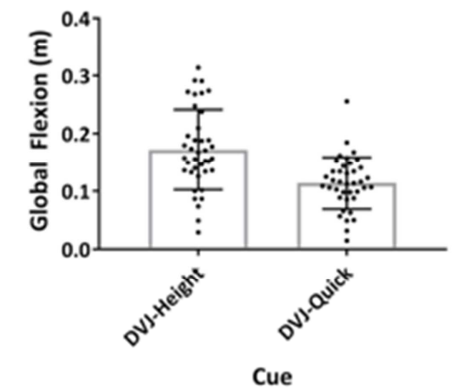

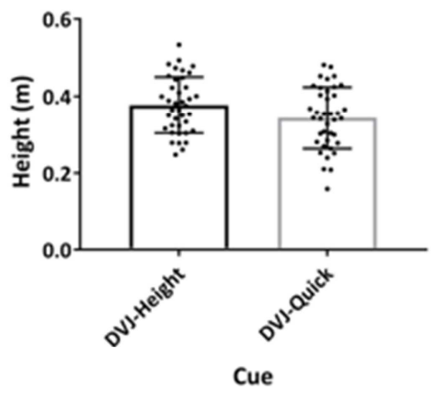

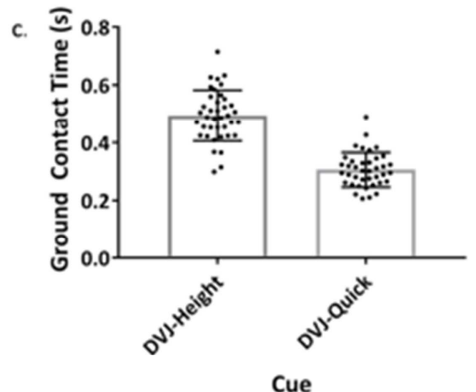

e.

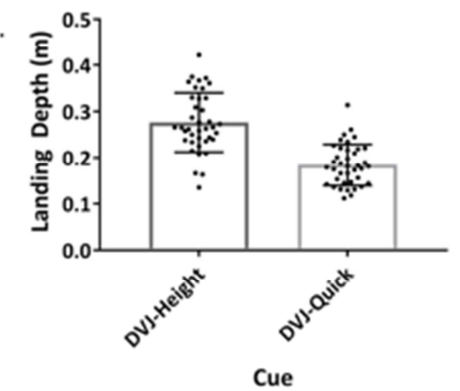

Figure 2. Bar charts of all dependent variables. For all measured variables, the $95 \%$ highest density intervals did not cross zero which is equivalent to $p<0.05$ in a frequentist context.

\section{Discussion \& Conclusion}

\subsection{Discussion}

The purpose of this study was to investigate the influence of cueing DVJs to maximize height or minimize ground contact time on landing mechanics and RSI. In confirmation of our hypothesis, RSI increased with large effect when performing a $\mathrm{DVJ}_{\text {Quick. }}$ Landing depth concurrently decreased with large effect, indicating a common solution was to increase landing stiffness for the $\mathrm{DVJ}_{\text {Quick }}$ technique. When cued to jump as high as possible without regard for time on the ground, subjects successfully increased jump height concurrent with increased landing depth. The secondary purpose was to investigate global flexion in the lower extremity joints at initial impact. In confirmation of our second hypothesis, the $\mathrm{DVJ}_{\text {Height }}$ cue resulted in more global flexion at impact. The $\mathrm{DVJ}_{\text {Quick }}$ cue still resulted in global flexion at impact but was not as substantial. These results suggest the $\mathrm{DVJ}_{\text {Quick }}$ cue leads to a stiffer landing due to earlier contact with the ground, and decreased vertical displacement of the center of mass during deceleration when compared to the $\mathrm{DVJ}_{\text {Height }}$ cue. These two measurements, global flexion at impact and vertical displacement during deceleration, compound on each other and result in decreased range of motion at the final eccentric position before concentric motion begins.

\subsection{Implications}

The implications of our results will vary depending on the situation, objective, and environment of testing. The type of 
verbal cue can have significant influences on performance [28]. For example, external cues (focus on the movement effect) often lead to improved motor skill performance and learning [28] compared to internal cues (focus on the body), but internal cues may lead to more muscle activation than external cues [28], suggesting a constrained and inefficient movement. The verbal cues in this study to "to jump as high as you can after landing" or "to get off the ground as quick as you can" were intended to be relatively neutral cues on the internal to external cue spectrum to be sure subjects understood the difference in objectives [4]. Given the large effects our cues had on jump performance and landing mechanics, coaches cueing plyometric exercises should pay careful attention to align their cues with the goal of training or testing. RSI and stiffness were reported to increase in youth athletes after just 4 weeks of plyometric training with cues to maximize jump height and minimize ground contact time [22]. A cue to minimize time on the ground is recommended if a practitioner wants to maximize RSI. Greater RSI was accomplished with less time (faster) and more stiffness (decreased landing depth), which could indicate a greater stretch-shortening cycle stimulus and tendon stretch [10]. Thus, a training program focused on maximizing the intensity of the stretch shortening cycle should focus on minimal ground contact time in both training and testing. In contrast, a cue to maximize jump height is recommended if a practitioner wants to decrease the intensity of the stretch-shortening cycle while performing plyometrics. This could be particularly useful as a late stage rehabilitation exercise prior to performing the DVJ for minimal ground contact time. In effect, these cues could be used to progress or regress plyometric intensities during rehabilitation, training, and testing. Regarding future research into the influence of verbal cues on plyometric performance, a comparison of external and internal cues could be useful for practitioners, in addition to a comparison of cues when external load is applied to movement.

It is especially important for practitioners to appreciate differences in cues if the DVJ is used as a testing or monitoring tool to direct training program decisions. For example, some training paradigms may use RSI to signal a regression or progression in performance and trigger modifications to exercise selection or training volume and intensity. However, a large decrease or increase in RSI could occur due to an athlete performing the test incorrectly, with inconsistent technique, or with submaximal effort. This makes RSI vulnerable to volatility. In these cases, unintended and inefficient periodization decisions could be made based on jump technique rather than jump performance. If coaches are using RSI to dictate or support periodization decisions, it is critical that athletes are performing the depth jump with consistent cues, techniques, and objectives.

One primary limitation of the current study is using ground reaction forces to estimate body positions at takeoff and landing. We have estimated global flexion in the hip, knee, and ankle joints at impact using the difference in COM displacement between impact and takeoff. It needs to be acknowledged that full extension of the hips, knees, and ankles may not occur at takeoff, and should be considered when interpreting the current dataset. However, we had no occurrences where the COM was higher at initial impact compared to takeoff, which indicates there is flexion of the lower extremity joints prior to initial impact. Regarding vGRF integration, this study anchored the velocity-time curve to 0 $\mathrm{m} / \mathrm{s}$ at mid-flight. However, we know the final landing positions could present increased lower extremity joint flexion relative to the DVJ takeoff position, which artificially increases flight time and consequently jump height. To avoid this methodological challenge, we could anchor the velocity-time curve to $0 \mathrm{~m} / \mathrm{s}$ at the final standstill, which has been demonstrated with good efficacy [13]. Unfortunately, we were unable to apply this method to our current dataset because not all trials had clear standstill motion following the final landing of the depth jump. Another consideration is the variations in jumping ability across subjects.

Despite subjects being on the same sporting team (men and women's soccer) with similar training programs, some subjects were in their first year of the program and may have not be as technically developed or comfortable with their DVJ techniques. Since data were only compared within each subject and trials were collected during the same session the statistical analysis should account for some of this variability. Further, generalizing our results to non-soccer athletes may result in different outcomes. Sports that incorporate more or less frequent jump training may find smaller or larger effects from verbal cues and objectives during the DVJ. However, the strong effect sizes observed in the current study would indicate some change is likely to occur in DVJ performance between cues for maximum height compared to quickness. Future studies could focus on athletes across different sports or skill levels.

\subsection{Conclusion}

RSI is a practical and effective measurement of jump performance during plyometric tasks, but the current study demonstrated how verbal cues and landing techniques have a strong influence on RSI during depth jumps. Cueing athletes to perform the DVJ to get off the ground as quickly as possible resulted in a large increase in RSI due to a disproportionate decrease in ground contact time (large decrease) compared to jump height (small decrease). The increase in RSI coincided with a decrease in landing depth, suggesting a stiff landing strategy and an increased stretch-shortening cycle intensity. To maximize RSI values, athletes should be cued to focus on minimizing ground contact time. Coaches should prioritize consistent verbal cues, techniques, and objectives during DVJ tests if RSI is used to support or dictate athlete readiness and periodization decisions.

\section{Funding Disclosure}

This research did not receive any specific grant from funding agencies in the public, commercial, or not-for-profit sectors. 


\section{Conflict of Interest Statement}

The authors declare that they have no competition interests.

\section{Acknowledgements}

The authors would like to thank the subjects for their participation in the study.

\section{References}

[1] Barker, LA, Harry, JR, and Mercer, JA. Relationships Between Countermovement Jump Ground Reaction Forces and Jump Height, Reactive Strength Index, and Jump Time. J Strength Cond Res 32 (1): 248-254, 2017.

[2] Beattie, K, Carson, BP, Lyons, M, Kenny, I. The Relationship Between Maximal Strength and Reactive Strength. Inter $J$ Sports Physiol Performance 12: 548-553, 2017.

[3] Beattie, K, Flanagan, EP. Establishing the Reliability \& Meaningful Change of the Drop-Jump Reactive-Strength Index. J Aust. Strength Cond 23 (5): 12-18, 2015.

[4] Benz, A, Winkelman, N, Porter, J, Nimphius, S. Coaching Instructions and Cues for Enhancing Sprint Performance. Strength and Conditioning Journal 38 (1): 1-11, 2016.

[5] Cormie, P, McBride, JM, and McCaulley, GO. Power-Time, Force-Time, and Velocity-Time Curve Analysis of the Countermovement Jump: Impact of Training. J Strength Cond Res 23 (1): 177-186, 2009.

[6] Cormie, P, McGuigan, MR, and Newton, RU. Changes in the Eccentric Phase Contribute to Improved Stretch-Shorten Cycle Performance after Training. Med Sci Sports Exerc 42: 17311744,2010

[7] Cronin, JB, Hansen, KT. Strength and Power Predictors of Sports Speed. J Strength Cond Res 19 (2): 349-357, 2005.

[8] Devita, P and Skelly, WA. Effect of landing stiffness on joint kinetics and energetics in the lower extremity. Med Sci Sport Exerc 24: 108-115, 1992.

[9] Douglas, J, Pearson, S, Ross, A, McGuigan, M. Kinetic Determinants of Reactive Strength in Highly Training Sprint Athletes. J Strength Cond Res 32 (6): 1562-1570, 2017.

[10] Earp, J, Newton, R, Cormie, P, Blazevich, A. Faster Movement Speed Results in Greater Tendon Strain during Loaded Squat Exercise. Frontiers in Physiology 7: 366, 1-12. 2016.

[11] Flanagan, EP, Galvin, L, Harrison, A. Force Production and reactive Strength Capabilities After Anterior Cruciate Ligament Reconstruction. Journal of Athletic Training 43 (3): 249-257, 2008.

[12] Guy-Cherry, D, Alanazi, A, Miller, L, Staloch, D, Ortiz-Rodriguez, A. Landing Styles Influences Reactive Strength Index Without Increasing Risk for Injury. Sports Medicine International Open 2: E35-E40, 2018.

[13] Harry, JR, Barker, LA, Eggleston, JD, Dufek, JS. Evaluating Performance During Maximum Effort Vertical Jump Landings. Journal of Applied Biomechanics 34: 403-409, 2018.
[14] Harry, JR, Blinch, J, Barker, L, Krzyszkowski, J, Chowning, L. Low-Pass Filter Effects on Metrics of Countermovement Vertical Jump Performance. J Strength Cond Res, In Press, 2020 .

[15] Holbrook, A. K., Peterson, H. D., Bianchi, S. A., Macdonald, B W., Bredahl, E. C., Belshan, M, Siedlik, J. A.. CD4(+) T cell activation and associated susceptibility to HIV-1 infection in vitro increased following acute resistance exercise in human subjects. Physiol Rep 7 (18): e14234, 2019.

[16] Horita, T, Komi, PV, Nicol, C, Kyrolainen, H. Interaction Between Pre-Landing Activities and Stiffness Regulation of the Knee Joint Musculoskeletal System in the Drop Jump: Implications to Performance. Eur J Appl Physiol 88: 76-84, 2002.

[17] Khuu, S, Musalem, L, Beach, TA. Verbal Instructions Acutely Affect Drop Vertical Jump Biomechanics- Implications for Athletic Performance and Injury Risk Assessments. J Strength Cond Res, 29 (10): 2816-26, 2015.

[18] Kijowski, KN, Capps, CR, Goodman, CL, Erickson, TM, Knorr, DP, Triplett, TN, et al. Short-term Resistance and Plyometric Training Improves Eccentric Phase Kinetics Jumping. J Strength Cond Res 29 (8): 2186-2196, 2015.

[19] Komi, P. Stretch-Shortening Cycle: A Powerful Model to Study Normal and Fatigued Muscle. Journal of Biomechanics 33 (10): 1197-1206, 2000

[20] Kruschke, J. K. Bayesian estimation supersedes the t test. J Exp Psychol Gen, 142: 573-603, 2013.

[21] Li, F, Newton, R, Shi, Y, Sutton, D, Ding, H. Correlation of Eccentric Strength, Reactive Strength, and Leg Stiffness with Running Economy in Well-Trained Distance Runners. $J$ Strength Cond Res 00 (00): 1-9, 2019.

[22] Lloyd, RS, Oliver, JL, Hughes, MG, Williams, CA. The Effects of 4-Weeks of Plyometric Training on Reactive Strength Index and Leg Stiffness in Male Youths. J Strength Cond Res 26 (10): 2812-2819, 2012.

[23] Oliver, JL, Barillas, SR, Lloyd, RS, Moore, I, Pedley, J. External Cueing Influences Drop Jump Performance in Trained Young Soccer Players. J Strength Cond Res 00 (00): 1-7, 2019.

[24] Sanchez-Sixto, A, McMahon, JJ, Floria, P. Verbal instructions affect reactive strength index modificed and time-series waveforms in basketball players. Sports Biomechanics 00: 1-11, 2021.

[25] Santello, M. Review of Motor Control Mechanisms Underlying Impact Absorption From Falls. Gait \& Posture 21: 85-94, 2005.

[26] Taylor, K, Chapman, DW, Cronin, JB, Newton, MJ, Gill, N. Fatigue Monitoring in High Performance Sport: A Survey of Current Trends. J. Aust. Strength Cond 20 (1): 12-23, 2012.

[27] Team, R. C. R: A language and environment for statistical computing. R Foundation for Statistical Computing, Vienna, Austria. 2019.

[28] Wulf, G. Attentional focus and motor learning: a review of 15 years. International Review of Sport and Exercise Psychology 6: 1, 77-104. 2012. 